Review

\title{
Immune Metabolism in Functional Aspects of the Brain
}

\author{
Elisa Gonzalez Cuevas and Nitya G. Chakraborty \\ Department of Medicine, University of Connecticut School of Medicine, \\ 263 Farmington Avenue, Farmington, CT 06032, USA
}

\author{
Article history \\ Received: 17-05-2019 \\ Revised: 15-06-2019 \\ Accepted: 10-12-2019 \\ Corresponding Author: \\ Nitya G. Chakraborty \\ Department of Medicine, \\ University of Connecticut \\ School of Medicine, 263 \\ Farmington Avenue, \\ Farmington, CT 06032, USA \\ Email: chakraborty@uchc.edu
}

\begin{abstract}
The brain interacts with the immune system in a highly regulated and restricted manner. Immune signals initiate pro- and anti-inflammatory activity in most body tissues in response to disease states and injury. An accumulation of inflammatory insults can disrupt immune metabolic homeostasis and give rise to pathology in sensitive areas. Here, we provide an overview of interactions between immune metabolism and the brain, with an emphasis on immune homeostatic regulation. We propose that an imbalance in immune activity can have a dose and time dependent effect on susceptible tissues. In the brain, this can contribute to neuronal injury and subsequent decline in function. We also discuss a specific pathology application involving the inflammation model of Alzheimer's Disease (AD). AD exemplifies the complexities of a disease etiology with multiple contributing factors, which has complicated the development of effective therapies. Patients could thus benefit from an individualized risk assessment and prevention approach that focuses on preservation of function and minimization of immune metabolic damage.
\end{abstract}

Keywords: Immune Metabolism, Neurodegeneratve Diseases

\section{Introduction}

In addition to the classic view of the role of the immune system in elimination of pathogens and injury repair, there has been a more recent focus on its signaling role across organ systems (Tchessalova et al., 2018). Biochemical signals from immune metabolism can convey information about general states such as disease, stress and energy status and trigger tissuespecific responses. For example, systemic interleukin-6 (IL-6) levels increase after exercise, which in the short term triggers metabolic pathways that help maintain local energy demand and initiate tissue repair (Hennigar et al., 2017). IL-6 levels also increase acutely after sleep deprivation (Walsh et al., 2019), but this response can become damaging over time. Chronic sleep deprivation is associated with higher circulating cytokines and thus longer systemic inflammatory states, which negatively affect susceptible tissues in the long term (Meerlo et al., 2009). This mechanism may be a contributor to the observed association between sleep deprivation and reduced hippocampal volume, as well as increased risk for cognitive and psychiatric disorders (Irwin et al., 2015). Overall, excessive accumulation of inflammatory cytokines in the CNS has been associated with memory and learning deficits. These cytokines, often released during times of stress or pathology, include tumor necrosis factor $\alpha$ (TNF- $\alpha)$ and interleukins

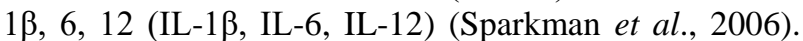
Interestingly, cytokine receptors are more concentrated in brain areas that are involved in learning and memory, which represents a direct impact of systemic homeostasis on brain function through immune metabolic activity.

Excessive inflammatory immune signaling can have detrimental effects, but the presence of activated immune cells in the brain is not exclusive to pathology. There are immune metabolic functions that have the capacity to prevent excessive inflammation and maintain proper brain function. Interleukin-4 (IL-4) is a key regulator of the inflammatory response in the brain and it has also been found to play a key role in learning and memory function (Gadani et al., 2012). The absence of IL-4 producing $\mathrm{T}$-cells in meningeal tissue has a negative impact on cognitive performance, perhaps through actions on astrocyte production of brain derived neurotrophic factor (BDNF) (Derecki et al., 2010). Decreased microglial responsiveness to IL-4 has also been shown to be associated with depressive-like behavior in a mouse model (Wachholz et al., 2017). Therefore, IL-4 may contribute to regulation of multiple brain functions not directly related to disease state, such as cognition, mood and affect. Cytokines as regulators of neuronal function is an understudied topic that has great potential to provide us with risk assessment and potential 
treatment avenues for neurocognitive disease. It is thus relevant to explore the regulation of passage of immune signals into the brain through the blood-brain barrier and the choroid plexus, as well as cellular mediators.

\section{Blood-Brain Barrier}

The Blood-Brain Barrier (BBB) encompasses the cells that form and surround the blood vessels of the central nervous system and that act as a filter from the systemic circulation. The BBB maintains homeostasis in the brain by regulating influx of nutrients and signals from other body systems, as well as efflux of metabolic waste. The cells that make up the BBB include specialized vascular endothelial cells, pericytes and astrocytes. Tight Junctions (TJ), Adherens Junctions (AJ) and gap junctions are transmembrane and membrane-associated cytoplasmic proteins that connect adjacent endothelial cells and thus provide the first physical barrier from the systemic circulation. TJs allow or impede passage into and out of the blood vessels in response to metabolic demand and cellular signaling (Luissint et al., 2012). These junctional proteins also provide an entryway for the systemic immune system through the regulation of passage of lymphocytes during inflammation (Stamatovic et al., 2016).

Other transport proteins in endothelial cells include solute-specific transporters such as solute carriermediated transporter and ATP-Binding Cassette (ABC) transporters. ABC transporters use energy from ATP to move molecules through the luminal endothelial cell membrane and are particularly abundant in the BBB, where they restrict the entry of drugs. Small molecules such as oxygen and carbon dioxide can pass directly through endothelial cells via rapid diffusion, thus maintaining the brain metabolic demands and a stable pH. (Zhao et al., 2015).

Endothelial cells share the same basement membrane with pericytes. Pericytes are tightly connected to the endothelium through $\mathrm{N}$-cadherin and connexins. Just like in the peripheral circulation, pericytes manage vascular permeability and passage of ions and metabolites, as well as release of growth factors that regulate angiogenesis. Importantly for immune function, pericytes regulate leukocyte transmigration through release of TGF- $\beta$ (Takeshita and Ransohoff, 2012). Pericytes are more densely packed in the brain than in the periphery and thus are essential for the integrity of the BBB. Loss of integrity of the BBB in older adults has been associated with neurodegenerative disease (Armulik et al., 2011). A recent study proposed an etiology for Parkinson's disease that involves alterations in the gut microbiota and a disruption of the gut- and blood-brain barrier causing increased permeability and abnormal accumulation of protein (Fitzgerald et al., 2019). Inappropriate immune stimulations and metabolic waste that accumulate over time can lead to metabolic disease etiologies that become more apparent with increasing age.
Another important component of the BBB are astrocytes, which are also the most abundant macroglia in the CNS. There are more astrocytes than neurons in the human brain cortex. Astrocytic feet surround endothelial cells and function to maintain the local environment homeostasis of neurons by regulating ion concentrations and neurotransmitter supply. In addition to these homeostatic functions, astrocytes also participate in information processing roles such as the formation and elimination of neuronal synapses and establishment of neuronal network patterns (Vasile et al., 2017). Astrocytes can act as immune modulating cells in the CNS and can trigger innate and adaptive immune responses. Antiinflammatory repair pathways are activated by astrocytes in response to an insult through secretion of protective factors and cytokines such as TGF- $\beta$, IFN- $\gamma$ and BDNF. Inflammatory factors induced by astrocytes include IL17, NFKB and VEGF. Dysfunction or inappropriate activation of these signaling pathways in astrocytes can lead to inflammation, demyelination and neuronal damage. The type of signaling pathways activation depends on the stimuli, extracellular environment and timing of the stimulus (Colombo and Farina, 2016). Additionally, in the brain ventricles, the choroid plexus functions as another filtrating barrier and the resulting filtrate that fills ventricular space is the Cerebrospinal Fluid (CSF), which provides cushioning and nutrients for the CNS. A recent study associated the age-related depletion of klotho protein in the choroid plexus to increased microglial activation and cytokine signaling in the hippocampus (Zhu et al., 2018). Microglia are the classic example of the resident macrophages in the brain and their role in immune function and neurodevelopment is discussed next.

\section{Microglia}

Microglia can initiate innate and adaptive immune responses. These cells are also involved in the maintenance of neuronal networks, as well as regulation of inflammation, phagocytosis of invaders, injury repair and remodeling. Microglia are derived from macrophages in the extra-embryonic yolk-sac. The role of microglia in neurodevelopment is crucial as they invade and seed the brain in early embryonic stages (Ginhoux et al., 2013). They have a direct effect on neuronal function and development, inducing neuronal differentiation, as well as modification of synaptic connections and pruning. Pruning is an important process that allows formation of efficient neural networks, as well as ongoing modification, which is the basis of learning and memory. Neurodevelopmental disorders, such as autism spectrum disorder and schizophrenia, have been associated with pruning imbalances (Hughes, 2012). In the brain, microglia exist in three forms based on immune activity: resting (ramified), activated, or phagocytotic. 
Resting microglia patrol the brain parenchyma, actively pruning "weak" connections and searching for invaders. Activated microglia can become phagocytotic and they express cytokines and MHC II. Microglia also express Fc receptors and are the only cell in the brain that express complement receptors.

Microglia activation has been described in terms of their macrophage polarization equivalent, i.e. "M1 microglia" are associated with classically inflammatory cytokines (IL-1 $\beta$, IL-6, IL-12, TNF- $\alpha$ ) and reactive oxygen species; and "M2 microglia" produce antiinflammatory cytokines and growth factors (Il-10, TGF- $\beta$, NGF, BDNF). However, this may be an oversimplified view of how immune responses work in the CNS in vivo as both responses are often present at the same time in the setting of neurodegenerative disease (Sarlus et al., 2017). Microglia are also important factors in the control of adaptive immune cells, such as T-cells and B-cells.

\section{T-Cells, Monocytes, Mast Cells}

Circulating leukocytes are able to infiltrate into the brain in response to injury and play an integral role in tissue repair. T helper 1, 2, 17 (Th1, Th2, Th17) and regulatory T-cells (Treg) can be found in the brain's choroid plexus, which acts as the entryway to the CSF for infiltrating T-cells and monocyte-derived macrophages in the context of injury or repair mechanisms. CD4+ effector memory Tcells regulate this entry of leukocytes through the epithelium by means of IFN- $\gamma$ signaling (Kunis et al., 2013). One common example is ischemic injury, where Tcells are the coordinators of the immune response which can trigger pro- and anti-inflammatory signals that have an effect on BBB integrity and function. T-cells have also been implicated in long-term tissue repair mechanisms and neurogenesis (Rayasam et al., 2017). Other cells implicated in injury repair are mast cells. Mast cells reside in the abluminal (brain) side of the BBB and recruit microglia when activated. As classical members of the innate immune system, mast cells are the "firstresponders". Their expression of mediators includes histamine, serotonin, IL-1 to IL-6, TNF- $\alpha$ and IFN- $\gamma$. (Dong et al., 2014). They are also the only hematopoietic cells that reside in the brain and usually become activated before microglia in the context of cerebral ischemia.

The direct impact of immune cells in the brain is the coordination of response to injury in an attempt to balance repair and detrimental inflammatory effects. The indirect interactions of immune metabolism and the brain, on the other hand, is a new area of study that can have more long-term effects in susceptible brain cells and their functions. T-cell auto-reactivity induced by bacteria in the gut has been associated with behavioral changes and may promote spatial learning and memory function (Wekerle, 2017). However, an excess of systemic T-cell activity has also been found to have detrimental effects on brain function and behavior. In a model of PD-1 deficiency, the associated excessive Tcell response resulted in amino acid depletion and consequent deficiency of neurotransmitters in the brain, which had an effect of increased anxiety and fear responses (Miyajima et al., 2017). Another study linked changes in social and anxiety-like behaviors in mice to consumption of specific dietary emulsifiers through the alteration of the intestinal microbiota, which gave rise to chronic low-grade inflammation and metabolic dysregulations (Holder et al., 2019). Thus, systemic immune signals can play a key role in regulation of proper brain function.

\section{Alzheimer's Disease: A Pathology Application}

The exact etiology of Alzheimer's Disease (AD) remains elusive after decades of investigation. $\mathrm{AD}$ is a progressive neurodegenerative disease that has been associated with the accumulation of extracellular amyloid$\beta(\mathrm{A} \beta)$ plaques in the brain parenchyma and intra-neuronal Tau-protein aggregates. It is still unclear whether this protein pathology is a direct cause of the disease, or a secondary marker that exacerbates disease progression. The single most associated risk factor for non early onset $\mathrm{AD}$ is increasing age. Other risk factors include genetics, family history, heart health, head injuries and hearing impairment. Lifestyle-related factors that increase risk include smoking and lower social engagement, whereas factors that decrease risk include use of statins, a Mediterranean diet, lightto-moderate alcohol consumption, higher educational attainment, physically and cognitively stimulating activities (Hersi et al., 2017).

The risk factors associated with $\mathrm{AD}$ suggest a variable pathogenesis that may be specific to each affected individual and which may be the result of biological or genetic risks that become expressed due to lifestyle and metabolic factors. The accumulation of these metabolic insults result in improper activation of immune cells, leading to inflammation and neurodegeneration. A study that used a metabolomics approach identified peripheral metabolic changes in patients with $\mathrm{AD}$ and Mild Cognitive Impairment (MCI). They found several changes in lipid metabolism earlier in the disease progression and suggested a pathophysiology involving defects in cell membranes that could lead to abnormal signaling, inflammation and neurodegeneration (Toledo et al., 2017). Multiple markers of inflammation and immune activation have also been found in AD patients' brains, such as cytokines, complement proteins and acute phase reactants (McCaulley et al., 2015). A Complement Receptor 1 (CR1) gene variant has been linked to an increased risk of $\mathrm{AD}$ development and thus complement-mediated microglia activation has been suggested as a contributor to the pathogenesis of AD (Sarlus et al., 2017). 
Furthermore, the most predictive gene variant for AD is Apolipoprotein E4 (APOE4), which is a protein involved in cholesterol transport and was recently found to cause higher innate immune reactivity in microglial cells in vitro after lipopolysaccharide exposure.

In this mouse model, APOE4 expression also resulted in higher TNF- $\alpha$ secretion and lower neuronal viability in the context of tau expression (Shi et al., 2017). Tau protein aggregation is an important component of pathology.

A mouse model found a causal relationship between glial senescence in cells expressing the cell cycle inhibitory protein $\mathrm{p} 16$, neurofibrillary tangle accumulation and cognitive decline. Importantly, in this study Tau aggregation and its cognitive effects were prevented with the action of senolytic agents (Bussian et al., 2018).

There are other proposed pathogeneses of AD, such as the impaired clearance of $A \beta$. Improper drainage of meningeal lymphatics may be a consequence of aging and a factor contributing to impaired $\mathrm{A} \beta$ clearance (Da Mesquita et al., 2018). The dysfunction of ABC transporters, important mediators of substance passage through the BBB, has also been associated with the disease (Deane et al., 2009; Zhao et al., 2015). Other proteins involved in $\mathrm{A} \beta$ clearance are low-density lipoprotein receptor related protein-1 (LRP1) and receptor for advanced glycation end products (RAGE). LRP1 regulates brain homeostasis through signaling pathways, including $A \beta$ metabolism and it responds to various ligands, such as apolipoproteins (Shinohara et al., 2017). Even hormonal regulation can have an impact on
A $\beta$ toxicity, as has been suggested by an association between increased serum thyroid levels and incidence of AD. Increased exposure to thyroid hormones imposes an excessive metabolic demand, which can lead to increased oxidative stress and thus vulnerability to metabolic byproduct toxicity (Quinlan et al., 2019).

Alzheimer's disease has a multifaceted pathophysiology that includes genetic predisposition, as well as lifestyle components that could be modifiable. Similar to cancer, there appears to be a pattern of "hits" that leads to an increasingly vulnerable state. These hits can be genetic, metabolic, immune inflammatory or a reduction in cognitive reserve. Early identification of modifiable lifestyle and health risk factors is important for attempts to delay or prevent disease onset, as shown in Fig. 1. This integrated approach includes addressing metabolic disorders that can trigger inflammatory pathways, such as diabetes, obesity and hyperlipidemia; as well as psychiatric mood disorders that reduce cognitive reserve, such as anxiety, depression (Manning et al., 2017), as well as their potential exacerbating but modifiable factors such as hearing loss (Rutherford et al., 2018). Risk reduction also involves maintaining adequate brain oxygenation and neuronal health, particularly in older adults, through regular exercise and engagement in cognitive and socially stimulating activities, to prevent accumulation of oxidative stress and early neuronal atrophy. These risk factors in combination with metabolic biomarkers may become a more active way to individualize prevention or even treatment strategies for cognitive decline and Alzheimer's disease.

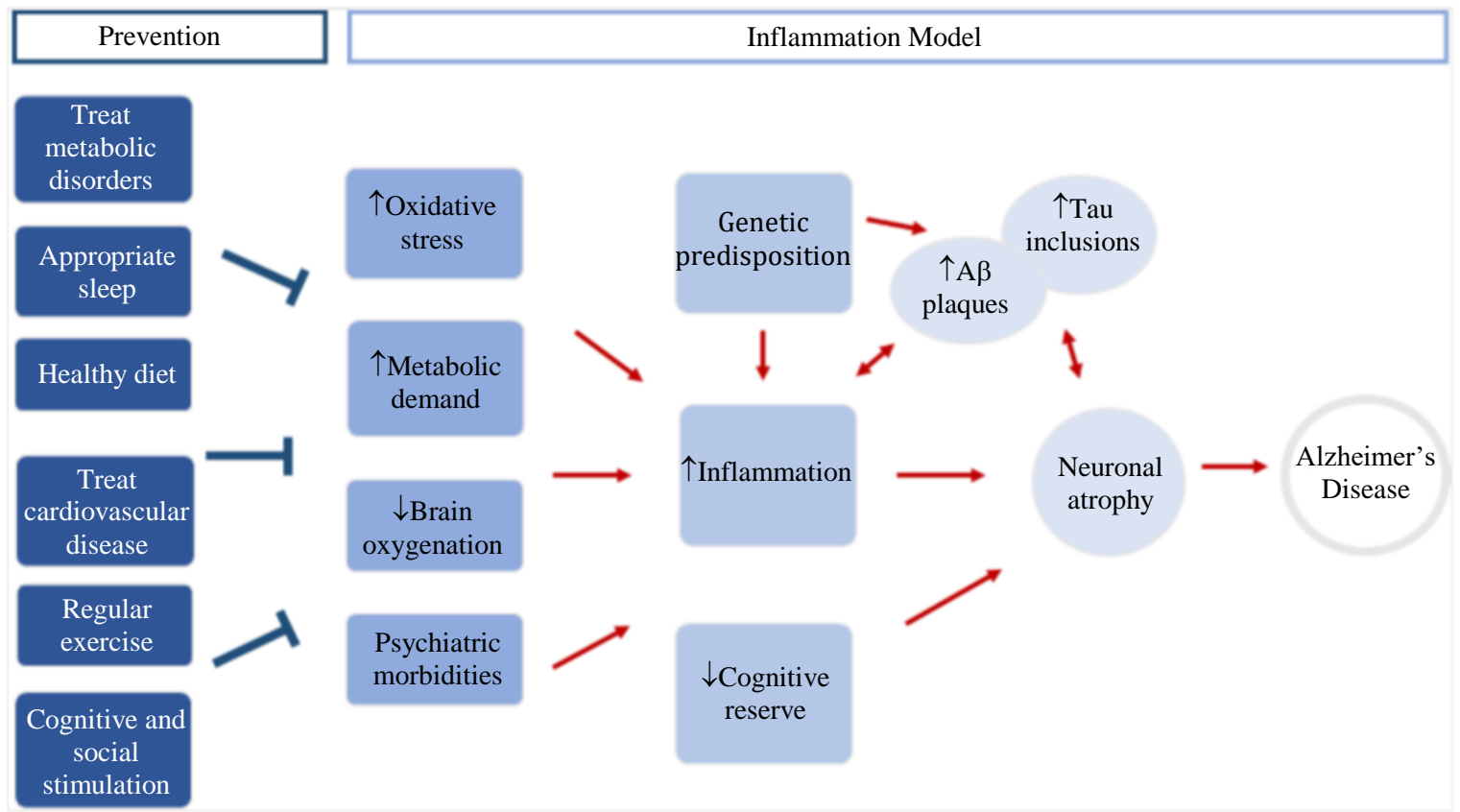

Fig. 1: Inflammation model and prevention measures for Alzheimer's disease 


\section{Conclusion}

Immune cells and cytokine activity in the brain were often strictly associated with pathologic states. However, research is shifting focus towards the beneficial effects of immune cells and their metabolites in the brain. The BBB is a key player in regulation of passage of immune cells and their signals. T-cells in the meninges and specific cytokines, such as IL-4, have a constructive effect in the brain by promoting learning and memory function. Inflammatory cytokines in excess generally have the opposite effect, but are necessary in the context of tissue injury and repair. An imbalance at any point in the immune activation pathway as a result of disruption of metabolic homeostasis can impact the normal function of brain cells. An example of how such an imbalance in immune metabolism may contribute to pathology is the inflammatory component of neurodegenerative pathologies, such as Alzheimer's disease as depicted in the Fig. 1.

In this connection it is very much appropriate to mention about the review article on immunometabolism by Wang et al. (2019) in recent issue of Science. They critically showed the reciprocal interactions between systemic metabolism and immunity. They also showed how (un controlled) inflammation could alter the functional state of various organs including brain. Eventually coordinated cross talk between whole-body metabolism and immune metabolism is involved in healthy and diseased states.

Advancement of medicine has significantly increased human life expectancy. Unfortunately, extended lifespan is associated with an increased incidence of certain diseases, including neurodegenerative diseases; and we have not yet done appropriate work on public health education. Chronic diseases linked to lifestyle factors are growing to epidemic proportions. Modern diet, high in calories, rich in sugars, fats, salt and food-additives, but low in complex carbohydrates, fiber, vitamins and minerals are the key risk factors. It is thus crucial to focus on preventative medicine and lifestyle changes that can reduce patient risk for immune metabolic imbalances and that can potentially avoid the development of the disease.

\section{Acknowledgment}

This work is dedicated to the memory of Dr. Chakraborty and his life-long passion for education.

Authors are thankful to the Department of Medicine at the University of Connecticut, School of Medicine for their full support of this article.

\section{Author's Contributions}

Elisa Gonzalez Cuevas: Contributed to the literature search, planning and writing of the paper, figure design, editing and revising.

Nitya G. Chakraborty: Contributed to the writing as well as the literature search, planning, editing and revising.

\section{Ethics}

All authors have read and approved this manuscript. We confirm that the content is original and that there are no conflicts of interest to report.

\section{References}

Armulik, A., G. Genové and C. Betsholtz, 2011. Pericytes: developmental, physiological and pathological perspectives, problems and promises. Dev. Cell, 16: 193-215. DOI: 10.1016/j.devcel.2011.07.001

Bussian, T.J., A. Aziz, C.F. Meyer, B.L. Swenson and J.M. van Deursen et al., 2018. Clearance of senescent glial cells prevents tau-dependent pathology and cognitive decline. Nature, 562: 578-582. DOI: 10.1038/s41586-018-0543-y

Colombo, E. and C. Farina, 2016. Astrocytes: Key regulators of neuroinflammation. Trends Immunol., 37: 608-620. DOI: 10.1016/j.it.2016.06.006

Da Mesquita, S., A. Louveau, A. Vaccari, I. Smirnov and R.C. Cornelison 2018. Functional aspects of meningeal lymphatics in ageing and Alzheimer's disease. Nature, 560: 185-191. DOI: $10.1038 / \mathrm{s} 41586-018-0368-8$

Deane, R., R.D. Bell, A. Sagare and B.V. Zlokovic, 2009. Clearance of amyloid-beta peptide across the blood-brain barrier: implication for therapies in Alzheimer's disease. CNS Neurol. Disord. Drug Targets., 8: 16-30.

Derecki, N.C., A.N. Cardani, C.H. Yang, K.M. Quinnies and A. Crihfield et al., 2010. Regulation of learning and memory by meningeal immunity: A key role for IL-4. J. Exp. Med., 10: 1067-80.

DOI: $10.1084 /$ jem. 20091419

Dong, H., X. Zhang and Y. Qian 2014. Mast cells and neuroinflammation. Med. Sci. Monit. Basic. Res., 20: 200-206. DOI: 10.12659/MSMBR.893093

Fitzgerald, E., S. Murphy and H.A. Martinson, 2019. Alpha-synuclein pathology and the role of the microbiota in parkinson's disease. Frontiers Neuroscince, 13: 369-369. DOI: $10.3389 /$ fnins.2019.00369

Gadani, S.P., J.C. Cronk, G.T. Norris and J. Kipnis, 2012. IL-4 in the brain: A cytokine to remember. J. Immunol., 189: 4213-4219. DOI: $10.4049 /$ jimmunol.1202246 
Ginhoux, F., S. Lim, G. Hoeffel, D. Low and T. Huber, 2013. Origin and differentiation of microglia. Front Cell Neurosci., 7: 45-45.

DOI: 10.3389/fncel.2013.00045

Hennigar, S.R., J.P. McClung and S.M. Pasiakos, 2017. Nutritional interventions and the IL-6 response to exercise. FASEB J., 31: 3719-3728.

DOI: 10.1096/fj.201700080R

Hersi, M., B. Irvine, P. Gupta, J. Gomes and N. Birkett et al., 2017. Risk factors associated with the onset and progression of Alzheimer's disease: A systematic review of the evidence. Neurotoxicology, 61: 143-187. DOI: $10.1016 /$ j.neuro.2017.03.006

Holder, M.K., N.V. Peters, J. Whylings, C.T. Fields and A.T. Gewirtz et al., 2019. Dietary emulsifiers consumption alters anxiety-like and social-related behaviors in mice in a sex-dependent manner. Sci. Rep., 9: 172-172.

DOI: $10.1038 / \mathrm{s} 41598-018-36890-3$

Hughes, V., 2012. Microglia: The constant gardeners. Nature, 485: 570-572. DOI: 10.1038/485570a

Irwin, M.R., T. Witarama, M. Caudill, R. Olmstead and E.C. Breen, 2015. Sleep loss activates cellular inflammation and Signal Transducer and Activator of Transcription (STAT) family proteins in humans. Brain Behav. Immun., 47: 86-92.

DOI: 10.1016/j.bbi.2014.09.017

Kunis, G., K. Baruch, N. Rosenzweig, A. Kertser and O. Miller et al., 2013. IFN- $\gamma$-dependent activation of the brain's choroid plexus for CNS immune surveillance and repair. Brain, 136: 3427-3440. DOI: 10.1093/brain/awt259

Luissint, A.C., C. Artus, F. Glacial, K. Ganeshamoorthy and P.O. Couraud, 2012. Tight junctions at the blood brain barrier: Physiological architecture and disease-associated dysregulation. Fluids Barriers CNS, 9: 23-23.

DOI: $10.1186 / 2045-8118-9-23$

Manning, K. J., G. Chan and D.C. Steffens, 2017. Neuroticism Traits Selectively Impact Long Term Illness Course and Cognitive Decline in Late-Life Depression. Am. J. Geriatr. Psychiatry, 25: 220-229. DOI:10.1016/j.jagp.2016.10.006

McCaulley, M.E. and K.A. Grush, 2015. Alzheimer's disease: Exploring the role of inflammation and implications for treatment. Int. J. Alzheimers Dis., 2015: 515248-515248. DOI: $10.1155 / 2015 / 515248$

Meerlo, P., R.E. Mistlberger, B.L. Jacobs, H.C. Heller and D. McGinty, 2009. New neurons in the adult brain: The role of sleep and consequences of sleep loss. Sleep Med. Rev., 13: 187-94. DOI:10.1016/j.smrv.2008.07.004
Miyajima, M., B. Zhang, Y. Sugiura, K. Sonomura and M.M. Guerrini, 2017. Metabolic shift induced by systemic activation of T cells in PD1-deficient mice perturbs brain monoamines and emotional behavior. Nat. Immunol., 18: 1342-1352.

DOI: $10.1038 /$ ni.3867

Quinlan, P., A. Horvath, A. Wallin and J. Svensson, 2019. Low serum concentration of free triiodothyronine (FT3) is associated with increased risk of Alzheimer's disease. Psychoneuroendocrinology, 99: 112-119.

DOI: 10.1016/j.psyneuen.2018.09.002

Rayasam, A., M. Hsu, G. Hernández, J. Kijak and A. Lindstedt et al., 2017. Contrasting roles of immune cells in tissue injury and repair in stroke: The dark and bright side of immunity in the brain. Neurochem. Int., 107: 104-116.

DOI: 10.1016/j.neuint.2017.02.009

Rutherford, B.R., K. Brewster, J.S. Golub, A.H. Kim and S.P. Roose, 2018. Sensation and Psychiatry: Linking agerelated hearing loss to late-life depression and cognitive decline. Am. J. Psychiatry, 175: 215-224.

DOI: 10.1176/appi.ajp.2017.17040423

Sarlus, H. and M.T. Heneka, 2017. Microglia in Alzheimer's disease. J. Clin. Invest., 127: 3240-3249. DOI: 10.1172/JCI90606

Shi, Y., K. Yamada, S.A. Liddelow, S.T. Smith and L. Zhao et al., 2017. ApoE4 markedly exacerbates taumediated neurodegeneration in a mouse model of tauopathy. Nature, 549: 523-527.

DOI: $10.1038 /$ nature24016

Shinohara, M., M. Tachibana and T. Kanekiyo, 2017. Bu G. Role of LRP1 in the pathogenesis of Alzheimer's disease: evidence from clinical and preclinical studies. J. Lipid. Res., 58: 1267-1281.

DOI: 10.1194/jlr.R075796

Sparkman, N.L., J.B. Buchanan, J.R. Heyen, J. Chen and J.L. Beverly, 2006. Interleukin-6 facilitates lipopolysaccharide-induced disruption in working memory and expression of other proinflammatory cytokines in hippocampal neuronal cell layers. J. Neurosci., 26: 10709-10716.

Stamatovic, S.M., A.M. Johnson, R.F. Keep and A.V. Andjelkovic, 2016. Junctional proteins of the bloodbrain barrier: New insights into function and dysfunction. Tissue Barriers., 4: e1154641. DOI: $10.1080 / 21688370.2016 .1154641$

Takeshita, Y. and R.M. Ransohoff, 2012. Inflammatory cell trafficking across the blood-brain barrier: Chemokine regulation and in vitro models. Immunol. Rev., 248: 228-239.

DOI: $10.1111 /$ j.1600065X.2012.01127.x 
Tchessalova, D., C.K. Posillico and N.C. Tronson, 2018. Neuroimmune Activation Drives Multiple Brain States. Front. Syst. Neurosci., 12: 39-39.

DOI: 10.3389/fnsys.2018.00039

Toledo, J.B., M. Arnold, G. Kastenmüller, R. Chang and R.A. Baillie et al., 2017. Alzheimer's Disease neuroimaging initiative and the alzheimer disease metabolomics consortium. Metabolic network failures in 18 Alzheimer's disease: A biochemical road map. Alzheimers Dement., 13: 965-984. DOI: $10.1016 /$ j.jalz.2017.01.020

Vasile, F., E. Dossi and N. Rouach, 2017. Human astrocytes: structure and functions in the healthy brain. Brain Struct. Funct., 222: 2017-2029.

DOI: $10.1007 / \mathrm{s} 00429-017-1383-5$

Wachholz, S., A. Knorr, L. Mengert, J. Plümper and R. Sommer et al., 2017. Interleukin-4 is a participant in the regulation of depressive-like behavior. Behav. Brain Res., 326: 165-172. DOI: $10.1016 /$ j.bbr.2017.03.020

Walsh, C.P., A. Lim, A.L. Marsland, R.E. Ferrell and S.B. Manuck, 2019. Circulating Interleukin-6 concentration covaries inversely with self-reported sleep duration as a function of polymorphic variation in the glucocorticoid receptor. Brain Behav. Immun., 78: 21-30.

DOI: 10.1016/j.bbi.2019.01.002
Wang, A., H. Luan and R. Medzhitov, 2019. An evolutionary perspective on immunometabolism. Sci., 363: 3932. DOI: 10.1126/science.aar3932

Wekerle, H., 2017. Brain autoimmunity and intestinal microbiota: 100 Trillion Game Changers. Trends Immunol., 38: 483-497. DOI: $10.1016 /$ j.it.2017.03.008.

Zhao, Z., A.R. Nelson, C. Betsholtz and B.V. Zlokovic, 2015. Establishment and dysfunction of the BloodBrain Barrier. Cell, 163: 1064-1078. DOI: $10.1016 /$ j.cell.2015.10.067

Zhu, L., L.R. Stein, D. Kim, K. Ho and G.Q. Yu, 2018. Controls the brainimmune system interface in the choroid plexus. Proc. Natl. Acad. Sci., 115: e11388-e11396. DOI: 10.1073/pnas.1808609115 